\title{
STABLE AUGMENTATION QUOTIENTS OF ABELIAN GROUPS
}

\author{
Alfred W. Hales
}

\author{
To the Memory of Ernst Straus
}

\begin{abstract}
Let $G$ be a finite abelian $p$-group, $\mathrm{Z} G$ the associated integral group ring, and $\Delta$ its augmentation ideal. This paper determines the stable structure of the augmentation quotients $\Delta^{n} / \Delta^{n+1}$ and the structure of the graded ring $\mathrm{gr} \mathrm{Z} G$. It also gives an application to the dimension subgroup problem, extending earlier results of Gupta-Hales-Passi.
\end{abstract}

1. Introduction. Let $\mathbf{Z} G$ be the integral group ring of a finite abelian group $G$. Denote by $\Delta$ the augmentation ideal of $\mathbf{Z} G$, i.e. the kernel of the map from $\mathbf{Z} G$ to $\mathbf{Z}$ sending each group element to 1 . Further denote by $Q_{n}$ the $n$th "augmentation quotient" $\Delta^{n} / \Delta^{n+1}$. Then Bachman and Grunenfelder [1] have shown that, for all $n \geq n_{0}=n_{0}(G)$, we have $Q_{n} \cong Q_{n+1} \cong Q_{n+2} \cdots$ as abelian groups. Let $Q_{\infty}=Q_{\infty}(G)$ denote the "eventual" isomorphism type of the $Q_{n}$. A number of papers ([2], [5], [6], [7], [10], [11], [12], [13], [15]) have been devoted to the determination of $Q_{\infty}(G)$ in terms of $G$. In [4] we gave a conjecture for the structure of $Q_{\infty}(G)$ and verified this conjecture whenever $G \cong\left(C_{p^{n}}\right)^{m}$ for some $m$ and $n$. Here we shall establish the truth of this conjecture for all finite abelian $G$, and in the process determine $n_{0}=n_{0}(G)$ and the structure of the graded ring gr $\mathbf{Z} G$ associated to $\mathbf{Z} G$. We also give an application (extending a result in [3]) to the dimension subgroup problem.

The reader should consult Passi [8] for general background on the subject, and [4] for more specific background on this problem.

2. Description of results. Without loss of generality we may assume that $G$ is a finite abelian $p$-group, in which case $Q_{\infty}$ is also easily seen to be such a group. One way of viewing our problem is that we wish to determine the invariants of $Q_{\infty}$ in terms of those of $G$. Instead, however, we give an explicit presentation of (a group isomorphic to) $Q_{\infty}$ from which the invariants of $Q_{\infty}$ can be determined in a straightforward (though tedious) manner.

Define an abelian group $Q_{G}$ via generators and relations as follows: let $P_{G}$ denote the poset of cyclic subgroups $H$ of $G$. (So $P_{G}$ is a tree with 
root $H_{0}=\{e\}$.) Take one generator $x_{H}$ for each $H \in P_{G}$, and relations $p x_{H}=x_{K}$ wherever $H$ covers $K$ in $P_{G}\left(\right.$ and the relation $x_{H_{0}}=0$ ). Then we have

THEOREM 1. Let $G$ be a finite abelian p-group. Then $Q_{G} \cong Q_{\infty}(G)$.

In [4] this theorem was proved in the homocyclic case, i.e. when $G \cong\left(C_{p^{n}}\right)^{m}$, for some $m$ and $n$. The proof involved the establishment of a collection of "two-variable" identities in $\mathbf{Z} G$. Here we establish a larger collection of identities, some involving more variables, which will suffice to prove our result. They will also enable us to determine $n_{0}(G)$ and to give a presentation for the graded ring $\operatorname{gr} \mathbf{Z} G$.

Our results will immediately yield, using techniques of [3], the following

THEOREM 2. Let $G$ be a finitely generated metabelian group so that $G / G^{\prime}$ has exponent $p^{k}$. Then the $(n+1)$ st term $\gamma_{n+1}$ in the lower central series coincides with the $(n+1)$ st dimension subgroup $D_{n+1}$ for all $n \geq$ $p^{k}+p^{k-1}$.

Although our Theorem 1 gives a very satisfactory description of $Q_{\infty}$, we regard its proof as far from satisfactory-being computational rather than conceptual. The difficulty seems to stem from the non-naturality of the isomorphisms guaranteed by Bachman and Grunenfelder.

3. Identities. We begin with the new two-variable identities, which are "unbalanced" forms of those established in [4]. These new identities were in fact stated in [4], but details of their proofs were omitted.

Let $G=\langle g\rangle \times\langle h\rangle$ where $g$ and $h$ have orders $p^{m}$ and $p^{n}$, respectively, with $m \geq n$. Denote by $x$ and $y$ the elements $g-1$ and $h-1$, respectively, of the group ring $\mathbf{Z} G$. (Here our notation differs somewhat from [4].) We will also work with the polynomial $\operatorname{ring} \mathbf{Z}[X, Y]$, which maps surjectively to $\mathbf{Z} G$ by substituting $x$ for $X, y$ for $Y$.

LEMMA 1. For each $k, 0 \leq k<n$, we have that

$$
p^{k} y^{(m-n)\left(p^{n-k}-p^{n-k-1}\right)}\left(x^{p^{n-k}} y^{p^{n-k-1}}-x^{p^{n-k-1}} y^{p^{n-k}}\right)
$$

lies in $\Delta^{(m-n)\left(p^{n-k}-p^{n-k-1}\right)+p^{n-k}+p^{n-k-1}+1}$.

Proof. The strategy of the proof is similar to that of Lemma 1 in [4]. We regard $m-n$ as fixed throughout, and first show how the $k=0$ case 
follows from the validity of all cases with larger $k$ (and the same $m, n$ ). Then we show how the $k=0$ case for given $m, n$ implies all cases $k=t$, $m^{\prime}=m+t, n^{\prime}=n+t$.

Consider in $\mathbf{Z} G$ the following product:

$$
\left(\prod_{i=0}^{p^{n}-1}\left(g h^{-i}-1\right)\right)\left(\prod_{i=1}^{m-n} \prod_{\substack{j=0 \\(j, p)=1}}^{p^{n}-1}\left(g^{p^{i}} h^{-j}-1\right)\right) \cdot\left(\prod_{i=0}^{p^{n-1}-1}\left(g^{-i p^{m-n+1}} h-1\right)\right) .
$$

Since any character of $G$ must annihilate one of the factors, this product is 0 in $\mathbf{Z} G$. Now, as in [4], we use the standard map from $G$ to $\Delta / \Delta^{2}$ to conclude that the product

$$
\left(\prod_{i=0}^{p^{n}-1}(x-i y)\right) \cdot\left(\prod_{i=1}^{m-n} \prod_{\substack{j=0 \\(j, p)=1}}^{p^{n}-1}\left(p^{i} x-j y\right)\right) \cdot\left(\prod_{i=0}^{p^{n-1}-1}\left(-i p^{m-n+1} x+y\right)\right)
$$

lies in $\Delta^{(m-n)\left(p^{n}-p^{n-1}\right)+p^{n}+p^{n-1}+1}$. We then apply Lemma 3 of [4] repeatedly to this latter product, observing that calculations $\bmod p^{n}$ are legitimate for any term involving $y$ since $p^{n} y \in \Delta^{2}$. Eventually we conclude that, when $p$ is odd, the element

$$
y^{(m-n)\left(p^{n}-p^{n-1}\right)}\left(x^{p} y-x y^{p}\right)^{p^{n-1}}
$$

lies in $\Delta^{(m-n)\left(p^{n}-p^{n-1}\right)+p^{n}+p^{n-1}+1}$. Expanding this by the binomial theorem we see that the two outer terms are the ones we want,

$$
y^{(m-n)\left(p^{n}-p^{n-1}\right)}\left(x^{p^{n}} y^{p^{n-1}}-x^{p^{n-1}} y^{p^{n}}\right) .
$$

The other terms, paired according to their distances from opposite ends, are formal multiples of instances of Lemma 1 with $k>0$, and as such are easily seen to lie in the appropriate power of $\Delta$. This concludes the $k=0$ case for odd $p$.

For $p=2$ the argument is similar but somewhat more tedious, as was the corresponding case in [4].

Suppose now that the $k=0$ case of Lemma 1 has been established for given $m, n$. We show how to deduce the $k=1, m^{\prime}=m+1, n^{\prime}=n+1$ case from this. (A similar argument will handle $k=t, m^{\prime}=m+t$, $n^{\prime}=n+t$.) Let

$$
f(X, Y)=Y^{(m-n)\left(p^{n}-p^{n-1}\right)}\left(X^{p^{n}} Y^{p^{n-1}}-X^{p^{n-1}} Y^{p^{n}}\right),
$$

and let $l=(m-n)\left(p^{n}-p^{n-1}\right)+p^{n}+p^{n-1}+1$. Then, from the $k=0$ case, we know that

$$
\begin{aligned}
f(X, Y)-u(X, Y)= & g(X, Y)\left((X+1)^{p^{m}}-1\right) \\
& +h(X, Y)\left((Y+1)^{p^{n}}-1\right)
\end{aligned}
$$


where $u$ has all terms of degree $\geq l$. To establish the $k=1, m^{\prime}=m+1$, $n^{\prime}=n+1$ case, i.e. to show $p f\left(x^{\prime}, y^{\prime}\right) \in\left(\Delta^{\prime}\right)^{l}$ when $x^{\prime}=g^{\prime}-1, y^{\prime}=$ $h^{\prime}-1$, and $g^{\prime}, h^{\prime}$ have orders $p^{m+1}, p^{n+1}$ respectively, it will clearly suffice to show that $p g\left(x^{\prime}, y^{\prime}\right)\left(\left(x^{\prime}+1\right)^{p^{m}}-1\right)$ and $p h\left(x^{\prime}, y^{\prime}\right)\left(\left(y^{\prime}+1\right)^{p^{n}}-1\right)$ lie in $\left(\Delta^{\prime}\right)^{l}$. Recall from [4], however, that $p\left(\left(x^{\prime}+1\right)^{p^{m}}-1\right)$ lies in $\left(\Delta^{\prime}\right)^{p^{m+1}-p^{m}+1}$ and that $p\left(\left(y^{\prime}+1\right)^{p^{n}}-1\right)$ lies in $\left(\Delta^{\prime}\right)^{p^{n+1}-p^{n}+1}$. For $p$ odd this immediately implies that $p g\left(x^{\prime}, y^{\prime}\right)\left(\left(x^{\prime}+1\right)^{p^{m}}-1\right)$ lies in $\left(\Delta^{\prime}\right)^{\prime}$, since $p^{m+1}-p^{m}+1 \geq l$. It is more difficult to handle

$$
p h\left(x^{\prime}, y^{\prime}\right)\left(\left(y^{\prime}+1\right)^{p^{n}}-1\right) \text {. }
$$

For this we consider the equation $(*)$ more carefully. If $h(X, Y)=$ $\sum_{l, j} a_{i j} X^{l} Y^{j}$, with $p^{e_{i j}}$ the highest power of $p$ dividing $a_{i j}$, then it suffices to show $\left(e_{i},+1\right)\left(p^{n+1}-p^{n}\right)+1+i+j \geq l$ for all $i, j$. Suppose not, and let (for a given $i$ ) $e_{\imath}$ be minimal so that this fails. Then the term $a_{i j} X^{i} Y^{j} Y^{p^{n}}$ occurs on the right hand side of $(*)$, and all other occurrences of $X^{i} Y^{j+p^{n}}$ in $(*)$ have coefficients divisible by a higher power of $p$ than

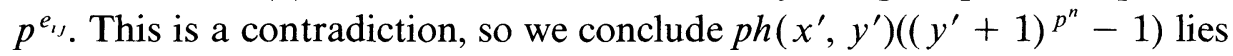
in $\left(\Delta^{\prime}\right)^{l}$ also. Hence $p f\left(x^{\prime}, y^{\prime}\right) \in\left(\Delta^{\prime}\right)^{l}$ when $p$ is odd. (The case $p=2$ is only slightly more complicated.)

This completes the proof of Lemma 1.

Now we pass to the multivariable identities. Let $G=\langle g\rangle \times\langle h\rangle \times$ $\left\langle a_{1}\right\rangle \times \cdots \times\left\langle a_{r}\right\rangle$, with $g, h$ as before and with $a_{i}$ having order $p^{e_{t}}$ for each $i$. Let $x=g-1, y=h-1$, and (for each $i$ ) $z_{i}=a_{i}-1$ in the group ring $\mathbf{Z} G$. Then the polynomial ring $\mathbf{Z}\left[X, Y, Z_{1}, \ldots, Z_{r}\right]$ maps surjectively to $\mathbf{Z} G$ in the obvious way. For any $k \geq 0$, denote by $\theta_{m, n, k}$ the following element of $\mathbf{Z} G$ :

$$
\theta_{m, n, k}=y^{(m-n)\left(p^{n-k}-p^{n-k-1}\right)}\left(x^{p^{n-k}} y^{p^{n-k-1}}-x^{p^{n-k-1}} y^{p^{n-k}}\right) .
$$

Then we have

Lemma 2. Suppose $n=e_{0}>e_{1}>e_{2}>\cdots>e_{r}$ and, for each $i$ with $1 \leq i \leq r$, that $k_{i}$ is a positive integer such that $e_{l}+k_{\imath} \leq e_{l-1}$. Suppose $k \geq 0$ and define, for each $i, d_{t}=e_{l}-\sum_{j=t+1}^{r} k_{j}-k$ and $s_{i}=p^{d_{t}-1}+$ $k_{i}\left(p^{d_{i}}-p^{d_{i}-1}\right)$. Also let $q=\sum_{i=1}^{r} k_{i}+k$. Then

$$
p^{k} \cdot \theta_{m, n, q} \cdot z_{1}^{s_{1}} \cdot z_{2}^{s_{2}} \cdots z_{r}^{s_{r}}
$$

lies in $\Delta^{\prime}$, where

$$
l=(m-n)\left(p^{n-q}-p^{n-q-1}\right)+p^{n-q}+p^{n-q-1}+\sum_{l=1}^{r} s_{l}+1 .
$$


Proof. Notice that, for $r=0$, this is just Lemma 1. The proof is in fact a considerably more elaborate version of the second half of the proof of Lemma 1. We consider first the case $k=0, p$ odd, and proceed by induction on $r$. More precisely, we work in the polynomial ring $R=$ $\mathbf{Z}\left[X, Y, Z_{1}, \ldots, Z_{r}\right]$ and assume that we have an identity

$$
\begin{aligned}
(* *) \quad Y^{(m-n)\left(p^{n-q}-p^{n-q-1}\right)}\left(X^{p^{n-q}} Y^{p^{n-q-1}}-X^{p^{n-q-1}} Y^{p^{n-q}}\right) Z_{1}^{s_{1}} Z_{2}^{s_{2}} \cdots Z_{r}^{s_{r}} \\
=d+A\left((X+1)^{p^{m}}-1\right)+B\left((Y+1)^{p^{n}}-1\right) \\
+\sum_{i=1}^{r} C_{i}\left(\left(Z_{i}+1\right)^{p^{e_{1}}}-1\right)
\end{aligned}
$$

such that

(1) $d \in(\Delta(R))^{l}$

(2) each element $A, B, C_{1}, \ldots, C_{r-1}$ lies in the ideal generated by $Z_{r}^{p_{r}-1}, p Z_{r}^{p_{r}-2}, \ldots, p^{e_{r}-1} Z_{r}$

(3) $B$ lies in the ideal generated by all $p^{e_{i j}} X^{i} Y^{j}$ with

$$
\begin{aligned}
\left(e_{i j}\right. & +1)\left(p^{n-q+1}-p^{n-q}\right)+1+i+j \\
& \geq(m-n)\left(p^{n-q}-p^{n-q-1}\right)+p^{n-q}+p^{n-q-1}+1 .
\end{aligned}
$$

(4) For $i=1,2, \ldots, r, C_{i}$ lies in the ideal $(\Delta(R))^{l_{c}}$ where

$$
l_{i}=(m-n)\left(p^{n-q}-p^{n-q-1}\right)+p^{n-q}+p^{n-q-1}+\sum_{j=1}^{i-1} s_{j} .
$$

Note that condition (1) guarantees that the assumed identity implies the corresponding case of Lemma 2. Furthermore, we have essentially verified that the case $r=0$ of this identity holds in the process of proving Lemma 1. (Indeed we were required to verify condition (3) in the second half of the proof of Lemma 1.)

We must now show that this identity implies the corresponding identity (a) for $k_{r}$ replaced by $k_{r}+1$ and each of $m, n, e_{1}, \ldots, e_{r-1}$ also increased by 1 , and also the corresponding identity (b) for $r$ replaced by $r+1$, with a new variable $Z_{r+1}$ where $k_{r+1}=1$ and $e_{r+1} \leq e_{r}-1$, and each of $m, n, e_{1}, \ldots, e_{r}$ increased by 1 .

Two facts about integral group rings of cyclic groups are used for this purpose. Suppose $a$ has order $p^{e+1}$, and $z=a-1$ in $\mathbf{Z}\langle a\rangle$. Then

(i) $p\left((z+1)^{p^{e}}-1\right) \in \Delta^{p^{e+1}-p^{e}}\left((z+1)^{p^{e}}-1\right)$

(ii) $z^{p^{e+1}}$ lies in the ideal generated by $p z^{p^{e}}, p^{2} z^{p^{e-1}}, \ldots, p^{e+1} z$.

The first of these was proved in [4] (and earlier in [9]), and was used implicitly in the second half of our Lemma 1 . The second of these is just the binomial theorem. 
Now, to derive identity (a), we multiply both sides of $(* *)$ by

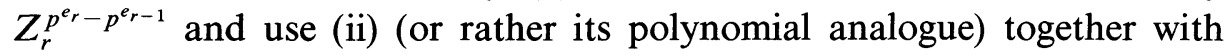
condition (2) to provide an extra factor of $p$ in each term (except the first) on the right-hand side. This factor of $p$ enables us, by (i), to replace each of these terms by a corresponding term (with $m, n$, or an $e_{i}$ increased by 1 ) plus a term which (by either condition (3) or (4)) lies in a high enough power of $\Delta(R)$ to be absorbed in the "new" $d$.

To derive identity (b), we proceed in a similar manner. We multiply both sides of $(* *)$ by $Z_{r+1}^{p^{e_{r+1}}}$ and use (ii) to provide a factor of $p$ in each term (except the first) on the right-hand side. Then, by (i), we can use this factor of $p$ to replace each of these terms by a corresponding term (with $m, n$, or an $e_{i}$ increased by 1) plus a term which can be absorbed in the new $d$. In the process a new term is introduced on the right-hand side involving $\left(Z_{r+1}+1\right)^{p^{e_{r+1}}}-1$, which is easily seen to satisfy the appropriate case of condition (4).

The verification that conditions (1)-(4) persist in each of cases (a) and (b) is straightforward.

This completes the proof of Lemma 2 for $k=0$ and $p$ odd. The proof of the second half of Lemma 1 can now be immediately adapted to handle higher values of $k$. Finally, the case $p=2$ can be handled in a similar but somewhat more complicated fashion.

Lemmas 1 and 2 give us "identities" which hold in the augmentation quotients $Q_{N}(G)$ for $N$ sufficiently large. For future use, note that whenever an identity from Lemma 2 holds for $G^{p}$, then $p$ times that identity holds for $G$. Furthermore, if such an identity holds for $G^{p}$, and all cyclic factors of $G^{p}$ have order $\geq p^{e}$, then $z^{p^{e}}$ times that identity holds for $G \times\langle a\rangle$, where $a$ has order $p^{e}$ and $z=a-1$. (Indeed, if such an identity holds for $G^{p^{t}}$ and all cyclic factors of $G^{p^{t}}$ have order $\geq p^{e}$, then $z^{p^{e-1}+t\left(p^{e}-p^{e-1}\right)}$ times that identity holds for $G \times\langle a\rangle$.)

4. Proof of Theorem 1. Singer showed in [14] that the order of $Q_{\infty}$ is $p^{c-1}$, where $c$ is the number of components of the rational group algebra $\mathbf{Q} G$. However, this number $c$ is also the number of cyclic subgroups of $G$. From this it is easy to see that $Q_{G}$ also has order $p^{c-1}$, so $\left|Q_{\infty}\right|=\left|Q_{G}\right|$. By the argument given in [4], it will be sufficient to show that, for any $k$ and for large $N, p^{k} Q_{N}$ can be generated by the number of elements required to generate $p^{k} Q_{G}$. This will force the (Ulm) invariants of $Q_{N}$ and $Q_{G}$ to coincide. (Note that the number of elements required to generate $p^{k} Q_{G}$ is the number of maximal cyclic subgroups of $G^{p^{k}}$.)

Let $G=\prod_{i=1}^{m}\left\langle g_{i}\right\rangle$, where $g_{l}$ has order $p^{e_{t}}$, and $e_{1} \geq e_{2} \geq \cdots \geq e_{m}$. For each $i$ let $x_{\imath}=g_{i}-1$ in $\mathbf{Z} G$. Then, for any $N, Q_{N}$ is generated by (the 
images of) all monomials $\prod_{j=1}^{m} x_{j}^{\alpha}$ with $\sum \alpha_{j}=N$. It will suffice to show that, for large $N$, there is a subcollection of these monomials which also generates $Q_{N}$ and whose cardinality is the number of maximal cyclic subgroups of $G$. (And to show a similar result for $p^{k} Q_{N}$ and $G^{p^{k}}$.) We proceed by induction on $m$, with the result being clear for $m=1$.

The maximal cyclic subgroups of $G$ can be partitioned into $e_{1}-e_{m}+$ 2 classes in the following way. For each $i, 0 \leq i \leq e_{1}-e_{m}$, let $G_{i}=$ $\Pi_{j=1}^{m_{t}}\left\langle g_{j}\right\rangle$ where $m_{i}<m$ is chosen maximal so that $e_{m_{t}} \geq e_{m}+i$. Then let $T$, be the set of maximal cyclic subgroups of $G$ of the form $\left\langle\left(g_{1}^{\beta_{1}}, \ldots, g_{m}^{\beta_{m}}\right)\right\rangle$ where $\left\langle\left(g_{1}^{\beta_{1}}, \ldots, g_{m_{i}}^{\beta_{m_{i}}}\right)\right\rangle$ is a maximal cyclic subgroup of $G_{i}^{p^{l}}$ and, for $m_{i}<j<m, g_{j}^{\beta_{j}}$ has order less than $p^{e_{m}}$. Finally let $S$ be the set of maximal cyclic subgroups $\left\langle\left(g_{1}^{\beta_{1}}, \ldots, g_{m}^{\beta_{m}}\right)\right\rangle$ where $g_{j}^{\beta_{j}}$ has order less than $p^{e_{m}}$ for all $j<m$. Then we have

$$
\left|T_{0}\right|=p^{e_{m}} \cdot\left(\text { number of maximal cyclic subgroups of } G_{0}\right) \text {, }
$$

for $1 \leq i \leq e_{1}-e_{m}$,

$$
\begin{aligned}
\left|T_{l}\right|= & \left(p^{e_{m}}-p^{e_{m}-1}\right) \cdot\left(p^{e_{m}-1}\right)^{m-m_{\imath}-1} \\
& \cdot\left(\text { number of maximal cyclic subgroup of } G_{i}^{p^{l}}\right)
\end{aligned}
$$

and

$$
|S|=\left(p^{e_{m}-1}\right)^{m-1} .
$$

It is clear that $T_{0}, \ldots, T_{e_{1}-e_{m}}, S$ partition the set of maximal cyclic subgroups of $G$.

Now we construct sets $C_{0}, C_{1}, \ldots, C_{e_{1}-e_{m}}, D$ of monomials representing elements of $Q_{N}$ in such a way that $\left|C_{i}\right|=\left|T_{i}\right|$ for all $i$ and $|D|=|S|$. If we can show that these monomials generate $Q_{N}$ we will be finished. (The $C_{i}$ and $D$ depend on $N$, though our notation does not indicate this. For large $N$ the monomials we construct will be formally distinct, but for small $N$ the sets $C_{i}, D$ may overlap.)

Let $C_{0}$ be the set of all monomials $\prod_{j=1}^{m} x_{j}^{\alpha_{j}}$ where $0 \leq \alpha_{m}<p^{e_{m}}$, $\sum_{j=1}^{m} \alpha_{j}=N$, and where $\prod_{j=1}^{m-1} x_{j}^{\alpha}$ is a monomial which is a member of a previously chosen set of generators for $Q_{N-\alpha_{m}}\left(G_{0}\right)$. (Since $G_{0}$ has rank less than $n$, and we are proceeding by induction on $n$, this is legitimate.) Similarly, for each $i$ with $1 \leq i \leq e_{1}-e_{m}$, let $C_{i}$ be the set of monomials $\prod_{j=1}^{m} x_{j}^{\alpha}$, where

$$
p^{e_{m}-1}+i\left(p^{e_{m}}-p^{e_{m}-1}\right) \leq \alpha_{m}<p^{e_{m}-1}+(i+1)\left(p^{e_{m}}-p^{e_{m}-1}\right),
$$

$\alpha_{j}<p^{e_{m}-1}$ for $m_{l}<j<m, \sum_{j=1}^{m} \alpha_{j}=N$, and $\prod_{j=1}^{m_{l}} x_{j}^{\alpha_{j}}$ is a monomial which is a member of a previously chosen set of generators for 
$Q_{N-\sum_{j-m_{t}+1}^{m} \alpha_{j}}\left(G_{i}^{p^{\prime}}\right)$. Finally, let $D$ be the set of monomials $\prod_{J=1}^{m} x_{J}^{\alpha_{j}}$ where $\alpha_{j}<p^{e_{m}-1}$ for $j<m$, and $\sum_{j=1}^{m} \alpha_{j}=N$.

For $N$ sufficiently large it is clear (by induction on $m$ ) that $\left|C_{l}\right|=\left|T_{l}\right|$ for all $i$, that $|D|=|S|$, and that the sets $C_{i}$ and $D$ are pairwise disjoint. (It will suffice to take $N=\left(p^{e_{m}}-1\right)+N_{0}$, where $N_{0}$ is large enough so that a corresponding result holds for $G_{0}$.)

To show that the (images of the) monomials in the $C_{l}$ and $D$ generate $Q_{N}$, suppose to the contrary that some monomial $\prod_{j=1}^{m} x_{j}^{\alpha_{j}}$ with $\sum_{j=1}^{m} \alpha_{j}=$ $N$ cannot be expressed as a linear combination (modulo $\Delta^{N+1}$ ) of monomials in the $C_{i}$ and $D$, and that the $m$-tuple $\left(\alpha_{1}, \ldots, \alpha_{m}\right)$ is lexicographically greatest with this property. Consider the exponent $\alpha_{m}$, and first suppose $\alpha_{m}<p^{e_{m}}$. By induction the monomial $\prod_{j=1}^{m-1} x_{j}^{\alpha_{j}}$ is (modulo $\left.\Delta\left(G_{0}\right)^{N+1-\alpha_{m}}\right)$ a linear combination of a previously chosen set of generators for $Q_{N-\alpha_{m}}\left(G_{0}\right)$. Multiplying by $x_{m}^{\alpha_{m}}$, we obtain an expression for $\prod_{j=1}^{m} x_{j}^{\alpha}$ as a linear combination (modulo $\Delta^{N+1}$ ) of elements of $C_{0}$. Secondly, suppose for some $i$ with $1 \leq i \leq e_{1}-e_{m}$ that

$$
p^{e_{m}-1}+i\left(p^{e_{m}}-p^{e_{m}-1}\right) \leq \alpha_{m}<p^{e_{m}-1}+(i+1)\left(p^{e_{m}}-p^{e_{m}-1}\right) .
$$

If any $\alpha_{j}$ with $m_{l}<j<m$ satisfies $\alpha_{,} \geq p^{e_{m}-1}$, Lemma 1 allows us to simultaneously replace $\alpha_{m}$ by $\alpha_{m}-\left(p^{e_{m}}-p^{e_{m}-1}\right), \alpha_{J}$ by $\alpha_{j}+\left(p^{e_{m}}-\right.$ $\left.p^{e_{m}-1}\right)$ without changing the monomial modulo $\Delta^{N+1}$. This contradicts the lexicographic maximality of $\left(\alpha_{1}, \ldots, \alpha_{m}\right)$, so we conclude $\alpha_{j}<p^{e_{m}-1}$ for $m_{i}<j<m$. Now the monomial $\prod_{J=1}^{m_{t}} x_{j}^{\alpha_{j}}$, considered as an element of $Q_{N-\sum_{j=m_{\mathrm{t}}+1}^{m} \alpha_{j}}\left(G_{i}^{p^{i}}\right)$, can be written as a linear combination of the (images of) a previously chosen set of generators by induction, using previous instances of Lemma 2. But then multiplication by $\prod_{j=m_{l}+1}^{m} x_{J}^{\alpha_{j}}$ converts this to an expression for $\prod_{J=1}^{m} x_{J}^{\alpha_{j}}$ as a linear combination (modulo $\Delta^{N+1}$ ) of elements of $C_{l}$. (See the comments at the end of Section 3.) Finally, suppose $\alpha_{m} \geq p^{e_{m}-1}+\left(e_{1}-e_{m}+1\right)\left(p^{e_{m}}-p^{e_{m}-1}\right)$. Then as above we conclude that $\alpha_{J}<p^{e_{m}-1}$ for all $j<m$, so $\prod_{J=1}^{m} x_{J}^{\alpha_{j}}$ lies in $D$.

This concludes the proof that $Q_{N}$ can be generated by a collection of monomials whose cardinality equals the number of maximal cyclic subgroups of $G$. To show that, for each $k \geq 1, p^{k} Q_{N}$ can be generated by a collection of monomials $p^{k} \prod_{j=1}^{m} x_{j}^{\alpha_{j}}$ whose cardinality equals the number of maximal cyclic subgroups of $G^{p^{k}}$, we proceed in an exactly similar fashion, using Lemmas 1 and 2 with this value of $k$ (see the comments at the end of Section 3). This completes the proof of Theorem 1.

Let $U=U_{N}$ denote the union of the sets of monomials $C_{i}$ and $D$. (Recall that, for small $N$, these may overlap.) Any linear relation between the (images of the) elements of $U_{N}$ in $Q_{N}$ can be converted to a similar relation between the elements of $U_{N+M}$ in $Q_{N+M}$ by multiplying by $x_{J}^{M}$ for 
an appropriate $j$, and then reducing terms in the result (increasing their exponents lexicographically) as in the proof above. (The "appropriate $j$ " is $m$ if a term actually appearing in the relation comes from $D$, and otherwise is determined inductively in a similar fashion.) This would contradict $Q_{N+M} \cong Q_{\infty}$ for $M$ large. Hence no relations not implied by Lemma 2 can hold among the $U_{N}$ (for any $N$ ), and similarly for the corresponding sets for $k \geq 1$. Hence, as in [4], we conclude

Corollary 1. $Q_{N}$ is isomorphic to $Q_{\infty}$ if and only if

$$
N \geq\left(e_{1}-e_{2}\right)\left(p^{e_{2}}-p^{e_{2}-1}\right)+p^{e_{2}-1}+\sum_{j=2}^{m}\left(p^{e_{j}}-1\right)
$$

Corollary 2. The graded algebra gr $\mathbf{Z} G=\mathbf{Z} \oplus Q_{1} \oplus Q_{2} \oplus \cdots$ associated to $\mathrm{ZG}$ is isomorphic to the quotient of the polynomial ring $\mathbf{Z}\left[X_{1}, \ldots, X_{m}\right]$ by the ideal generated by all $p^{e_{i}} X_{i}(i=1, \ldots, m)$ and all homogeneous binomials given by application of Lemma 2 .

5. Proof of Theorem 2. By Corollary 2 of the previous section, the kernel of the map from $\mathbf{Z}\left[X_{1}, \ldots, X_{n}\right]$ to gr $\mathbf{Z} G$ is generated by a collection of homogeneous polynomials of degree at most $\left(e_{1}-e_{2}\right)\left(p^{e_{2}}-p^{e_{2}-1}\right)+$ $p^{e_{2}}+p^{e_{2}-1}$. (It is easy to see that this bound is minimal.) Hence, in the notation of [3], we have $n_{0}(G) \leq p^{e_{1}}+p^{e_{1}-1}=p^{k}+p^{k-1}$ where $k$ is the exponent of $G$. By Theorem 4.1 of [3] we thus obtain our Theorem 2. In fact we obtain the slightly stronger

THEOREM 2'. Let $G$ be a finitely generated metabelian group such that $G / G^{\prime}$ is a product of cyclic groups of orders $p^{e_{1}}, \ldots, p^{e_{m}}$ with $e_{1} \geq \cdots \geq e_{m}$. Then the $(n+1)$ st term $\gamma_{n+1}$ in the lower central series coincides with the $(n+1)$ st dimension subgroup $D_{n+1}$ for all $n \geq\left(e_{1}-e_{2}\right)\left(p^{e_{2}}-p^{e_{2}-1}\right)+$ $p^{e_{2}}+p^{e_{2}-1}$.

\section{REFERENCES}

[1] F. Bachman and L. Grunenfelder, The periodicity in the graded ring associated with an integral group ring, J. Pure and Appl. Algebra, 5 (1974), 253-264.

[2] M. Goyal, The augmentation ideal of an integral group ring, Ph.D. thesis, Kurukshetra University, Kurukshetra (India) 1981.

[3] N. D. Gupta, A. W. Hales and I. B. S. Passi, Dimension subgroups of metabelian groups, J. Reine Angew. Math., 346 (1984), 194-198.

[4] A. W. Hales, Augmentation terminals of finite abelian groups, in Abelian Group Theory (Proc. Honolulu, 1982-83), Springer-Verlag Lecture Notes \#1006, (1983), pp. 720-733. 
[5] K. Horibe and K. Tahara, The stable behavior of the augmentation quotients of some groups of order $p^{4}$, I, Japanese J. Math., 10 (1984), 137-157.

[6] G. Losey and N. Losey, The stable behavior of the augmentation quotients of the groups of order $p^{3}$, J. Algebra, 60 (1979), 337-351.

[7] I. B. S. Passi, Polynomial maps on groups, J. Algebra, 9 (1968), 121-151.

[8] _ Group Rings and Their Augmentation Ideals, Springer-Verlag Lecture Notes in Math., Vol. 715, Berlin-New York, 1979.

[9] R. Sandling, Modular augmentation ideals, Proc. Camb. Phil. Soc., 71 (1972), 25-32.

[10] M. Singer, On the graded ring associated with an integral group ring, Comm. in Algebra, 3 (1975), 1037-1049.

[11] __, On the augmentation terminal of a finite abelian group, J. Algebra, 41 (1976), $196-201$.

[12] _ Determination of the augmentation terminal for all finite abelian groups of exponent 4, Comm. in Algebra, 4 (1976), 639-645.

[13] _ Determination of the augmentation terminal for all finite abelian groups of exponent 8, Comm. in Algebra, 5 (1977), 87-100.

[14] _ Determination of the augmentation terminal for finite abelian groups, Bull. Amer. Math. Soc., 83 (1977), 1321-1322.

[15] K. Tahara and T. Yamada, The stable behavior of the augmentation quotients of some groups of order $p^{4}$. II, Japanese J. Math., 10 (1984), 159-184.

Received October 22, 1984.

UNIVERSITY OF CALIFORNIA

LOS ANGELES, CA 90024 